\title{
APUNTE LINGÜÍSTICO SOBRE LA PALABRA “MINACCIA” Y SU HISTORIA
}

\author{
A LINGUISTIC NOTE \\ ON MINACCIA (“THREAT”) AND ITS HISTORY
}

\author{
Paola Capponi \\ Universidad Pablo de Olavide, UPO, Sevilla, Espanha
}

\begin{abstract}
Resumen: En este artículo se ha elegido como hilo conductor la palabra MINACCIA "amenaza", palabra-clave en el discurso intimidatorio, que define una práctica antigua orientada a producir miedo en el destinatario. El objetivo es visibilizar rasgos antiguos de la percepción de la amenaza y contribuir así al estudio del discurso intimidatorio y de sus raíces. Más en concreto, se desarrolla un recorrido estrictamente lingüístico en torno a la palabra y a su historia, acudiendo principalmente a fuentes primarias y extendiendo puntualmente el análisis a la familia de palabras relacionadas con MINACCIA. Se detectan así los rasgos semánticos principales, variantes e invariantes.
\end{abstract}

Palabras-clave: amenaza; semántica; etimología; intimidación.

Abstract: This paper offers an analysis of the word MINACCIA "threat", a key-word for the study of discourse related to intimidation. The term refers to the practice (known from ancient times) of inducing fear in the addressee. Based mostly on primary sources, the paper reconstructs the history of the term by exploring both learned and popular traditions and including data about the family of words related to MINACCIA. The paper aims to contribute to the research in this field by highlighting some of the defining features of the word, pointing out semantic variants and invariants.

Key words: threat; semantics; etymology; intimidation.

\section{Introducción}

En este número se plantea la cuestión del origen y el devenir del discurso intimidatorio. La historia reciente nos dibuja un marco que exige una reflexión atenta y precisa sobre los límites, las características y las consecuencias del uso de la palabra y del discurso intimidatorio, el cual, cuando se reproduce exponencialmente en red y se difumina su autoría, da lugar a "hechos" de un nuevo alcance. El propósito de los estudios aquí compilados es indagar sobre el alcance de esta novedad, a saber: en qué medida se trata "solo" de un cambio de medios e instrumentos de medición o, sin embargo, de un cambio de tal magnitud y calado que exigen nuevas 
definiciones y nuevos instrumentos que permitan hacer un seguimiento y comprender el fenómeno. Es un tema que despierta indudablemente el interés de investigadores de distintas disciplinas (desde el derecho a la lingüística forense), cuyos estudios se centran en definir y medir el objeto de estudio así como en buscar rasgos y características que permitan delimitarlo.

En este breve estudio, de carácter puramente lingüístico, se escoge la palabra-clave MINACCIA (amenaza) y se reconstruye su historia y su espectro semántico: los posibles orígenes y su evolución atendiendo a la tradición culta y popular. Minaccia es palabra y práctica ancestral: se basa en anunciar una desgracia futura y, por tanto, infundir miedo y temor en el destinatario. Se puede expresar con palabras, gestos, silencios; va cargada a menudo de reticencia y mensajes implícitos; y, para que exista y produzca efecto, requiere una amplia cooperación interpretativa por parte del interlocutor. Sirva a modo de ejemplo el virgiliano Quos ego (Eneida I, p. 133-135), cuya expresión de la amenaza es fuertemente elíptica ${ }^{1}$.

La minaccia es objeto de estudio en lingüística, especialmente en el ámbito de la pragmática y de la lingüística forense, pero también de otras disciplinas que van de la psicología al derecho o a la filosofía ${ }^{2}$. Presenta un carácter particularmente escurridizo, tan solo reconocible e identificable en contexto, en el entramado de una serie compleja de variantes, una casuística potencialmente infinita de combinaciones contextuales. Esta dificultad no es nueva. Se reproducen, a título de ejemplo, las reflexiones ottocentescas de Pierre Fontanier con relación, precisamente, a la compleja definición en este caso retórica - de la amenaza. Fontanier (1977, p. 13), el Linneo de la retórica - en expresión de Genette, tomada de Balzac: "Linné de la rhétorique" -, incluye la amenaza, la commination, entre las supuestas figuras de pensamiento:

La commination est la menace ou l'annonce d'un malheur plus ou moins horrible, par l'image duquel on cherche à porter le trouble et l'effroi dans l'âme de ceux contre qui l'on se sent animé par la haine, la colère, l'indignation ou la vengeance (FONTANIER, 1977, p. 434).

\footnotetext{
${ }^{1}$ El motivo del Quos ego tuvo una notable repercusión iconográfica: "El modelo dado por el grabador italiano Marcantonio Raimondi en su estampa Quos Ego tuvo una gran repercusión en la iconografía de los esmaltes limusinos, como demuestran, entre otras obras, los retablos esmaltados del Museo Británico y del Museo Lázaro Galdiano”. Rodríguez López, 2004, p. 45. Véase la figura 1.

${ }^{2}$ Cabe señalar que se trata de un campo de estudio que está experimentando un mayor desarrollo en la actualidad, sobre todo en lo que respecta a las cuestiones relacionadas con la mejora de la prevención y de la seguridad.
} 
Siguen tres ejemplos extraídos de otras tragedias ${ }^{3}$ que al autor le plantean el interrogante sobre cuál es la especificidad de esta figura - «Seraitce donc l'objet particulier du langage, ou le sentiment, la passion que le langage exprime, qui ferait ici la figure?" - (FONTANIER, 1977, p. 435) y comenta:

Mais alors, autant de nouvelles figures, que de sentiments ou de passions diverses, ou que de diverses manières dont les sentiments, les passions peuvent éclater. Alors, dis-je, l'injure, le reproche, le blâme, le louange, la flatterie, le compliment, le conseil, l'exhortation, l'offre, la demande, le remerciement, la plainte, et que sais-je encore, seront autant de figures qu'il faudra classer sans doute d'après leurs caractères distinctifs de malignité et de violence, ou de douceur et d'aménité? (FONTANIER, 1977, p. 435).

De forma específica, sobre la amenaza escribe:

Et supposé que la Commination, la menace, pût n'être que feinte et simulée de la part de celui qui l'emploie, en quoi serait-elle plutôt une figure, qu'une promesse mensongère et perfide, ou que toute ruse quelconque, employée, pour tromper, subjuguer ou séduire ? Enfin, ne faudrait-il pas toujours, pour produire son effet et inspirer la terreur, qu'elle parût réelle et sérieuse, et ne pût pas être prise pour un vain jeu? (FONTANIER, 1977, p. 435).

En la introducción, Gérard Genette, en referencia a la categoría de supuestas figuras de pensamiento en la que Fontanier incluye la amenaza, explica:

Des mouvements de pensée tels que la délibération, la concession, l'interrogation, l'apostrophe, le souhait, la menace, etc., ne méritent le nom de figure que pour autant qu'ils se révèlent à l'analyse fictifs et artificiels. Poser une question [...] ne constitue pas en soi-même une figure: c'est une attitude de pensée qui s'exprime de manière adéquate et immédiate dans une tournure syntaxique. Pour trouver une figure dans une interrogation, il faut et il suffit d'y voir une "fausse interrogation", c'est-à-dire, de lire cette interrogation comme valant pour une assertion. (FONTANIER, 1977, p. $11)$.

Más recientemente, Noille (2014), ante el interrogante «formes du discours ou figures de pensées?», explica así la complejidad de la cuestión:

\footnotetext{
${ }^{3}$ Athalie, III, 1 (1691) y Andromaque I, 4 (1667) de Racine y L'orphelin de la Chine V, 4 (1755) de Voltaire.
} 
Un exemple suffira à comprendre le problème : l'expostulatio (ou reproches envers un ami) de même que la comminatio (ou menace) sont des genres discursifs comprenant un certain nombre de séquences hiérarchisées entre elles, dont les séquences focales que sont les formulations proprement dites $\mathrm{du}$ reproche ou de la menace. Mais ces mêmes formulations peuvent alors figurer à titre de séquences auxiliaires dans le canevas d'un autre genre, l'objurgation. Autrement dit, une comminatio est à la fois un genre du discours et une partie. Et à ce titre, une continuité syntagmatique existe entre un certain nombre de parties des discours et les figures de pensées : c'est ainsi que la menace et la prière sont mises en série avec d'autres formes, l'imprécation, l'optation, le serment, la promesse, la dubitation, la licence, etc., pour constituer la liste des figures de pensées ${ }^{4}$.

Cabe recordar, además, que la comminatio se clasifica como una "figura macroestructural de segundo nivel, es decir, un lugar" en AquienMolinié (1996, p. 95).

Así pues, el propósito específico de este estudio no es observar "la amenaza en acción" (cómo se produce, quiénes intervienen, los contextos, las relaciones de poder y las jerarquías entre los interlocutores, etc.) sino observar la palabra y su definición tal y como nos ha legado la tradición en el patrimonio lexicográfico. Lo que se propone es indagar sobre la percepción de la amenaza, sobre los rasgos semánticos relevantes que la tradición lexicográfica recoge, transmite y conserva. De este modo, se pretende contribuir a una reflexión lingüístico-cultural de un mayor alcance sobre la definición y la percepción de la amenaza. En esta línea, la investigación paremiológica ya ha aportado algunas indicaciones. Cuando los proverbios hacen referencia a la amenaza (el investigación se circunscribe a esta palabra y a sus derivadas, así pues, se excluyen otras expresiones sinónimas), utilizan algunos temas recurrentes que sirven para trazar una imagen de lo que se ha estado codificando como amenaza. Aislar variantes e invariantes, mediante estudios realizados desde una perspectiva diacrónica, contribuye a entender la continuidad dentro del cambio: una clave de lectura importante para comprender el discurso intimidatorio actual.

\footnotetext{
${ }^{4} \mathrm{Y}$ prosigue: «Car dans la mesure où la figure de pensées ne relève pas uniquement d'un fonctionnement sémiotique, mais possède une assise matérielle, une extension syntagmatique, et qu'elle est à proprement parler une figure, une forme qui modèle et délimite un lieu dans le fil discursif, elle est susceptible d'isoler un bloc d'énoncé, une séquence, au même titre que les séquences discursives dont nous parlons ici. Nous dirons qu'entre la figure de pensées et la séquence mise en forme dans un canevas discursif, la différence n'est pas d'ordre syntagmatique mais fonctionnel : la séquence discursive joue un rôle dans l'édifice général de la partition discursive, contrairement à la figure de pensées, qui n'a qu'une incidence locale» (NOILLE, 2014, p. 3). En su artículo, Noille propone tres lecturas del discurso de Germánico a las legiones romanas (Tácito): como "discours de réconciliation (conciliatio)" siguiendo la clasificación de Vossius (1621); "discours d'objurgation (objurgatio)" siguiendo la clasificación de Pelletier (1641) y "discours de menace (comminatio)" según la propia lectura.
} 


\section{Minaccia}

MINACCIA, en sus distintas formas (ant. manàcia, manaza; dialect. manazza, menaza, minaza, minazza), es la "presentación [...] de un mal futuro e injusto, cuyo cumplimiento dependerá de la voluntad de la misma persona que anuncia dicho mal". Ese anuncio de un mal futuro se puede «hacer expresamente, mediante palabras o gestos, o tácitamente, mediante un determinado comportamiento». Esta definición procede del Grande Dizionario della Lingua Italiana (GDLI), pero si leemos la primera acepción de la palabra MINAE en el Thesaurus Linguae Latinae (ThLL) encontraremos igualmente: "Actio minandi, denuntiatio mali verbis, gestibus, factis".

Mināciae, que ya usara Plauto (Miles gloriosus, v. 374), deriva del adjetivo minācem, "minaccioso" [amenazador] (en italiano además de minaccioso, encontramos minace, voz arcaica y desusada), a su vez derivado del verbo mināri, "elevarse, pender sobre algo o alguien" (DELI': s.v.). De mināciā, recuerda el ThLL: s.v., derivan también - además de la italiana minaccia- menace en francés, menassa en provenzal, amenaza en español y ameaça en portugués.

Las primeras referencias en italiano se remontan al siglo XIII. Encontramos "minaccia" en el poema Della caducità della vita umana (v. 43: manace); lo utiliza Guittone d'Arezzo (XCV, v. 9: minaccia) y aparece en Proverbia super natura feminarum (v. 426, 455, 458: manace; v. 677: manaça). Amminaccia (incluida la forma amminazo), derivada de minaccia, aparece por primera vez en los Dialoghi di san Gregorio de Giovanni Campulu (1302/37), con la acepción de "palabra o gesto mediante el que se declara una acción malévola hacia alguien a quien se pretende inducir que haga o deje de hacer algo". A esto se une el significado de "presagio de una desgracia", ya presente en la Istoria di Eneas, de Angelo di Capua $(1316 / 1337)^{6}$.

El italiano minacciare es un verbo denominativo antiguo, que quizás provenga del latín hablado *minaciāre, que aparece por primera vez con la forma manatiāre en las glosas de Reichenau (750 ca.): MINATUR: MANATIAT (KLAUSENBURGER, 2000, p. 40)7. En el Trecento

\footnotetext{
${ }^{5}$ Dizionario Etimologico della Lingua Italiana (CORTELAZZO; ZOLLI, [1979] 1988).

${ }^{6}$ La istoria di Eneas vulgarizzata per Angilu di Capua, ed. G. Folena, Palermo, 1956, 1. 6: 102.5, en: Tesoro della Lingua Italiana delle Origini - TLIO.

${ }^{7}$ DELI: s.v.: "Minnaciare es el verbo denominativo de minaccia (aunque la formación puede ser muy antigua, hasta tal punto que se podría pensar incluso en el latín hablado *minaciāre, que se documentó por primera vez con la forma manatiāre en las glosas de Reichenau, 750 ca.: Minatur: manatiat)".
} 
encontramos amminacciare (en sus distintas formas, entre las cuales amminazari), derivada de minacciare, de nuevo en los Dialoghi di san Gregorio di Giovanni Campulu (1302/1337) ${ }^{8}$. El significado va desde "infundir temor con palabras o gestos al objeto de inducir a que alguien haga o deje de hacer algo" hasta "lanzar amenazas". El adjetivo amenazivilli remite a amminacciare, "que expresa una amenaza, hostil", usado en el Factorum et dictorum memorabilium de Valerio Máximo traducido por Accurso di Cremona $(1321 / 37)^{10}$.

\section{Minacciare, menare, comminare}

El verbo minor, àris significa específicamente "amenazar»: «specialisé dans le sens moral de «menacer»; m. mortem alicuì (proprement "suspendre la mort sur quelqu'un")» (ERNOUT; MEILLET, I967: s.v. ${ }^{11}$. Como ya se ha indicado, el adjetivo mināx, -ācis remite a minor, y de dicho adjetivo deriva el sustantivo popular minācia(e), que posteriormente sustituyó a minae (ERNOUT; MEILLET, 1967: s.v.). La tradición docta y erudita del antiguo minari se mantiene y conserva mediante comminare (lat. comminari) 'requerir a alguien so pena de sanción' que apareció por primera vez (con la forma comminati) en las Constituciones egidianas de $1357^{12}$. Comminazione (con las formas cominatione, cominazione, comminacione, comminatione), en el sentido de 'requerir so pena de sanción', se registró también por primera vez en las Constituciones egidianas de 1357. Por otra parte, el significado religioso de 'amonestación, amenaza (de castigo)' aparece en las Prediche de Giordano da Pisa, $1309^{13}$.

\footnotetext{
${ }^{8}$ Libru de lu Dialagu de Sanctu Gregoriu traslatatu pir frati Iohanni Campulu de Missina, ed. S. SANtAngelo, Palermo 1933, 1. 4, cap. 46: 173.27, en: TLIO.

${ }^{9}$ A estos se añade el valor sustantivo. Cfr. Libru de lu Dialagu de Sanctu Gregoriu traslatatu pir frati Iohanni Campulu de Missina, ed. S. Santangelo, Palermo, 1933, 1. 2, cap. 23: 63.21), en: TLIO.

${ }^{10}$ Valeriu Maximu translatatu in vulgar messinisi per Accursu di Cremona, ed. F. A. UGolini, Palermo, 1967/1, 1. 1, cap. 6: 44.31, en: TLIO.

${ }^{11}$ Véase también immineō, sinónimo de īnstō, impendeō que significa «être situé ou suspendu au-dessus; dominer, menacer, être imminent» (ERNOUT; MEILLET, 1967: s.v.).

12 Colliva, P., Il Cardinale Albornoz, lo Stato della Chiesa, le "Constitutiones Aegidianae» (1353-1357), Boloña, 1977, 1. VI, cap. 9: 702.17, en: TLIO. Rezasco, G., Dizionario del linguaggio italiano storico ed amministrativo, Florencia 1881, s.v. COMMINARE: "amenazar con una pena a los transgresores de una ley u orden”, en: TLIO.

${ }^{13}$ Prediche del Beato F. Giordano da Rivalto dell'Ordine de' Predicatori, ed. D.M. Manni, Florencia, 1739, 33: 243.8, en: TLIO.
} 
A la lengua vulgar y popular pertenece la forma activa minō, -āre con el significado de 'conducir animales' ("Le conducteur les menac(s)ant de ses cris, de son fouet, etc."): "De mināri 'amenazar' se pasa de hecho a menare 'conducir', con la acepción 'conducir animales amenazándoles o golpeándoles', que resulta clara en la glosa de Festos: 'Agasones: equos agentes id est minantes' (p. 23 Lindsay)" (MIGLIORINI, 1994, p. 44). Así se explica no solo menare (ant. minare) "conducir", sino también el romanesco menare, "golpear, pegar", referido originariamente a las ovejas (DELI, s.v.). El verbo sirvió de base, como elemento de significado genérico, para la formación de locuciones populares. Se indican a continuación algunas: menarla per le lunghe "alargar la duración de algo" (antes de 1348), menare le mani "pegar" (s. XIV), menare la lingua "hablar maledicencias" (s. XIV), menare la coda "mover la cola" (s. XIV), menare il can per l'aia "dar largas a un asunto, darle vueltas sin llegar a una conclusión" (antes de 1565), menare le gambe "huir" (antes de 1589).

Menare per il naso qualcuno, "engañar o burlarse de alguien" (Domenico Cavalca, antes de 1342) es la expresión análoga de la francesa mener quelqu'un par le (bout du) nez (1559) que hace referencia a la costumbre de conducir al ganado bovino tirando de una cuerda atada a una nariguera. Así, Francesco D’Ambra, en Cofanaria IV 15, escribía:

$$
\begin{aligned}
& \text { m'hanno aggirato come un arcolajo, } \\
& \text { e menato pel naso come un bufalo } \\
& \text { [me han enredado como una rueca } \\
& \text { y tirado de la nariz como un búfalo] }
\end{aligned}
$$

De ahí que el verbo haya adoptado igualmente el significado de "engañar". En la actualidad, cubre un área semántica tan amplia y genérica que se puede utilizar también con un sentido obsceno (GALLI DE' PARATESI, 1964, p. 111).

La voz ammenare remite igualmente a menare y fue utilizado por primera vez por Pier della Vigna (1249) $)^{14}$. El primer significado es "conducir; traer, llevar; acompañar (t. fig.)" (en el sentido de "presentar en el lugar del juicio"; en relación con una sentencia de una autoridad o una alegación: "aducir"; y con referencia a las armas: "alzarse") ${ }^{15}$, con las

\footnotetext{
${ }^{14}$ Rime, en Poeti del Duecento, ed. G. Contini, Milán-Nápoles, 1960/1, 119-28: 123, en: TLIO.

${ }^{15}$ Cabe señalar que con el significado de 'aducir' se trata de un "galicismo semántico [...] que
} 
siguientes acepciones: "descargar, asestar" un golpe; "adelantarse, avanzar" $y$, en particular, ammenare contra, "enfrentarse (mediante amenaza)"; "seguir adelante, proceder". El segundo significado es "golpear, pegar". Con el significado de "enfrentarse a alguien mediante amenaza" se registró por primera vez en el Statuto del Comune e del Popolo di Perugia $(1342)^{16}$.

\section{Minae}

Mina, hoy día, es una voz culta y poco usada en italiano para minaccia (cabe señalar también el adjetivo minatorio, del lat. tardío minatōrium) ${ }^{17}$. En el ThLL, minae es una voz autónoma, distinta de mina ${ }^{18}$. En el Appendix Probi (IV.195, 26 GLOS.II 234, 2), además del primer significado, ya mencionado, análogo por completo a los diccionarios recientes ("Actio minandi, denuntiatio mali verbis, gestibus, factis"), presenta un segundo significado: "prominentia, pinnae murorum, cacumina montium (plerumque sensu metum incutiendi coniuncto"). Por tanto, en primer lugar aparece el sentido figurado de "amenaza" y en segundo lugar el significado físico, casi orográfico, de objetos prominentes, altos, dominantes y que, ya se especifica, infunden temor. En Ernout y Meillet (1967: s.v.) el primer significado de minae es "saillie, avance d'un mur, d'un rocher, surplomb" y se explícita el paso de "cosas suspendidas sobre" a "amenazas": "du sens de 'choses suspendues sur', on est passé au sens de 'menaces".

Con referencia a altura, reforzado mediante el prefijo, en italiano encontramos eminente "que sobresale respecto al ambiente circundante" y de ahí eminentissimo, eminenza, y también eminenza grigia, todas voces cultas de eminënte $(m)$, de eminère "sobresalir, elevarse", compuesto de ĕx y minnae, con la derivada eminĕntia $(m)$. Ėmineō es sinónimo de excellō

proviene de un intermediario perdido a través del cual pasa el lat. Adducere”, en: TLIO.

${ }^{16}$ Statuto del Comune e del Popolo di Perugia del 1342 in volgare, ed. M. S. Elsheikh, Perugia, 2000/2: 124.1, en: TLIO.

${ }^{17}$ En dialecto emiliano, minàcia significa 'paresia' ("a gh'é dä' na minàcia”, "sufre paresia”) o "infarto" ("tirèr da minàcia", "sufrir un infarto"). Se trata de una "italianismo con el sentido de "advertencia" de una desgracia aún mayor". Malaspina, C., Vocabolario parmigiano-italiano, Parma, 1856-1859 en: Dizionario Etimologico dei Dialetti Italiani - DEDI: s.v.

${ }^{18}$ Mina, homógrafo, es una unidad de peso equivalente a 100 dracmas aproximadamente (unos 430 gr.). Del gr. mnâ, de origen semítico. Véase el siguiente juego de palabras en Truculentus de Plauto (v. 948): "melius est minis certare mecum quam minaciis". Sobre el uso del plural cf. DELI: s.v.: "El uso solamente del plural en latín (con excepción de san Agustín) explica el uso análogo en italiano, hasta el punto que los teóricos (Buonmattei) consideraban que el uso del singular era inexistente en los buenos escritores (D’Alb.)". 
con el significado "se détacher en saillie, s'élever hors de (souvent au sens moral), d'où èminentissimus uir». Las voces también cultas prominente $\mathrm{y}$ prominenza, compuestas de prō, presentan una derivación análoga, con la misma formación que eminère (DELI: s.v.).

La etimología de minae no está clara (ERNOUT; MEILLET, I967: s.v.). A partir de la raíz indoeuropea, Grandsaignes d'Hauterive, en el Dictionnaire des racines des langues européennes (1949), identifica tres elementos esenciales. La raíz ${ }^{*}$ MIN- ${ }^{*}$ MON- indicaría: idea de elevación, altura; idea de montaña (mons, promontorium, y *montare); idea de nuca, crin, a menudo emparentada con algo que se eleva (sánscrito mányā "nuca", latín monìle, inglés mane "crin", alemán Mähne, "crin") ${ }^{19}$.

Ernout y Meillet, en el Dictionnaire étymologique de la langue latine (1967), señalan que la reconstrucción etimológica es incierta ("Aucune étymologie n'apparaît pour une forme minae, qui supposerait une racine *MEI-") y plantean una hipótesis que recupera la relación con ēmineō y con mōns: el hápax minent de Lucrecio no basta para aseverar la existencia de mineō y se podría pensar en una derivación de ēmineō, prōmineō, que son en cambio formas usuales ${ }^{20}$. Esta etimología, por tanto, remitiría a la raíz *MEN-, "être saillant", en latín representado por el ya mencionado mōns y por monile, quizás también por mentum ${ }^{21}$. Minae retomaría la raíz ${ }^{*} \mathrm{M}^{\mathrm{O}} \mathrm{N}-$ para posteriormente arribar a ${ }^{*} \mathrm{MIN}-$. En todo caso, concluyen: "pareille hypothèse est arbitraire» (ERNOUT; MEILLET, 1967: s.v.).

Recientemente el Etymological Dictionary of Latin (2008) confirma dichos valores. Michiel De Vaan retrotrae el origen de minae a *MIN- o a *MEN- (que se convierte en MIN- cuando no se encuentra en posición inicial, como en è-min-ēre). La preferencia que se da a esta derivación se apoya en la relación que se puede establecer entre è-min-ēre, mōns y mentum ${ }^{22}$.

19 Para la voz monīle, De Vaan (2008: s.v.) sugiere el proto-itálico ${ }^{*}$ MONJO- y el protoindoeuropeo *MON-I-, "cuello". En cuanto a la raíz, concluye: "Since there existed a productive pattern of deriving -li- adj. with a preceding long vowel from short-vowel $\mathrm{i}$ - and $\mathrm{u}$ - stems (LEUMANN, 1977, p. 350), monile can be derived directly from a preform *moni- 'neck'. In that case, the absence of unrounding to *MA- is unexpected. If the PIE i-stem was thematized to Pit. *MONI/o-, the first syllable was closed, and *MO- would be retained".

${ }^{20}$ Cf. De Vaan (2008: s.v.): "Lucr. Minēre is a recent back-formation to ē-min-ēre".

${ }^{21}$ Según Ernout y Meillet (1967) la voz monīle significa originariamente "nuca”. La noción de "nuca" y "objeto emergente, prominente" parecen guardar relación.

${ }^{22}$ De Vaan (2008: s.v) indica como significados de minae: "threats; protruding parts of Wall". De minae, derivan minārī, mināx, mināciae, comminārī, ēminārī, ēminātiō, interminārī, minitāre/̄̄, minitābiliter, ēminēre, èminulus, minēre. "In theory, these forms could reflect * MINor a stem ${ }^{*} \mathrm{MEN}$ - which changed to -min- in non-initial syllable, and was thence imported into minae and minārī. The fact that ē-min-ēre can be connected with mentum and mōns makes 


\section{Conclusiones}

El estudio del discurso intimidatorio parte de la intrínseca dificultad que conlleva definir el objeto de estudio. Es objeto igualmente de análisis de distintas disciplinas que trabajan en la elaboración de definiciones en función de los objetivos de los diferentes campos de investigación. De forma paralela, en la "lengua común", desde la antigüedad, el uso de las palabras empleadas para intimidar ha adquirido nombres y definiciones. Aquí analizamos una de ellas, MINACCIA, que representa notablemente uno de los elementos fundamentales de este tipo de discurso. En lugar de buscar los rasgos distintivos de minaccia a partir de un corpus de casos, se traza una imagen reconstruyendo el espectro semántico en torno a la raíz de la palabra, al objeto de observar los núcleos de significado ligados a esta palabra que, hoy día y de forma diversa, pueden estar presentes en la conciencia del hablante. El estudio del espectro semántico, de sus variaciones, pone de relieve en particular la idea del peligro que se cierne desde arriba, la idea del poder que, desde lo alto, infunde temor e induce una respuesta, provoca una reacción. Parece remitir a una situación espacial y, en paralelo, a una jerarquía de poder. Posteriormente, se subrayan como elementos focales la referencia al temor provocado y a la acción: palabra y acción, palabra y acto.

the second solution more attractive. Thus, the root ${ }^{*} \mathrm{MEN}-$ 'to stick out, protrude' is preserved in stative è-min-ēre. We may assume minae $<^{*} \mathrm{MEN}-\overline{\mathrm{A}}-\mathrm{meaning}$ first 'protruding part' and then 'threat', and from minā- was derived minārī. The verb served as the basis for mināx and for frequentative minitāre”. 


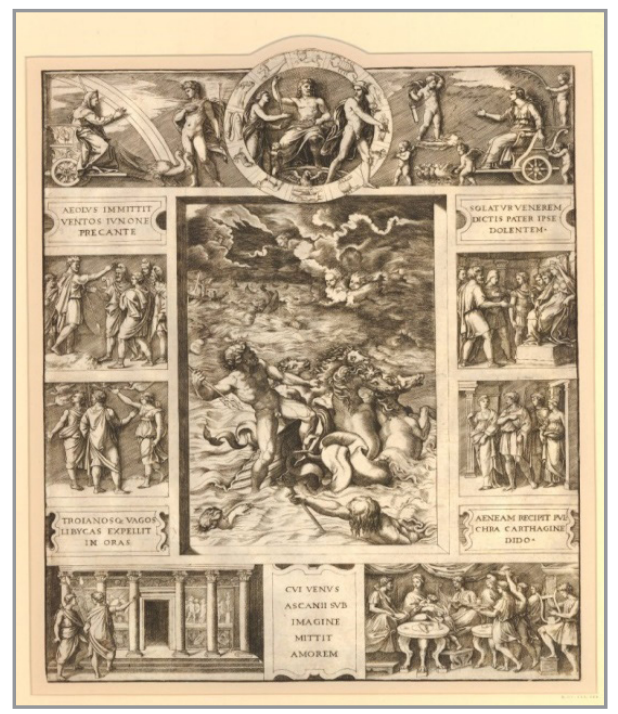

Figura 1: Quos ego

Fuente: British Museum, Museum number 1910,0212.340. (C) Trustees of the British Museum.

Descripción: Neptune calming the tempest which Aeolus raised against Aeneas' fleet in central compartment surrounded by nine scenes from Book I of the Aeneid Engraving

Comentario del curador: Traditionally known as the "Quos Ego" (being a quotation from Virgil), this print illustrates episodes from Book I of the Aeneid, and is inspired by the famous Tabula Iliaca (Capitoline) with Homeric scenes (here transposed to Virgilian scenes). According to Vasari, the lost original was drawn by Raphael in 1516 specifically to be translated into print format. Preliminary studies by Raphael exist at Chatsworth (Devonshire Collection, inv. 727a,b,c). Additional information: Part of the composition and figures of this print, in particular, the scene in the lower register, were used in an Italian maiolica bowl (British Museum,1855,1201.97); see D.Thornton and T.Wilson (Italian Renaissance Ceramics: a Catalogue of the British Museum Collection, vol. I, London, 2009, p. 251-253, cat. n.152).

\section{Referencias}

AQUIEN, Michèle; MOLINIÉ, Georges. Dictionnaire de rhétorique et de poétique. París: LGF, 1996.

CONTINI, Gianfranco. La letteratura italiana delle origini. Florencia: Sansoni, 1996.

DE VAAN, Michiel. Etymological Dictionary of Latin. Leiden-Boston: Brill, 2008. 
CORTELAZZO, Manlio; MARCATO, Carla. Dizionario Etimologico dei Dialetti Italiani. Turín: UTET, [1992] 2005.

CORTELAZZO, Manlio; ZOLLI, Paolo. Dizionario Etimologico della Lingua Italiana. Bolońa: Zanichelli, 1979-1988.

ERNOUT, Alfred; MEILLET, Antoine. Dictionnaire étymologique de la langue latine. París: Klincksieck, 1967.

FONTANIER, Pierre. Les figures du discours. Paris: Flammarion, 1977.

GALLI DE' PARATESI, Nora. Semantica dell'eufemismo. Turín: Giappichelli, 1964.

BATTAGLIA SALVATORE [después Giorgio BÀRBERI SQUAROTTI] (Org.). Grande dizionario della lingua italiana, 21 v., Turín: UTET, [1961] 2002.

GRANDSAIGNES D'HAUTERIVE, Robert. Dictionnaire des racines des langues européennes. Larousse: París, 1949.

KLAUSENBURGER, Jürgen. Grammaticalization: Studies in Latin and Romance morphosyntax. Amsterdam: J. Benjamins, 2000.

MIGLIORINI, Bruno. Storia della lingua italiana. Milán: Rizzoli, 1994.

NOILLE, Christine. Les genres du discours dans l'ancienne rhétorique : listes, schémas et mode d'emploi, avec un exemple (le discours de Germanicus). Exercices de rhétorique, n. 3, 2014. Disponible en: <http:// rhetorique.revues.org/337>. Acceso en: 01 dic. 2017.

RODRÍGUEZ LÓPEZ, M.I. Quos Ego. La transmisión iconográfica de la historia de Dido. Goya: Revista de arte, n. 298, p. 45-54, 2004.

Thesaurus Linguae Latinae, Leipzig, 1900 ss.

Tesoro della Lingua Italiana delle Origini. Disponible en: $<$ http://tlio.ovi. cnr.it/TLIO/>. Acceso en: 01 dic. 2017. 